\title{
Efeitos da ginástica aeróbica nas variáveis antropométricas de idosos
}

Effects of aerobic gymnastics on anthropometric variables of elderly

LIMA, F. É. B. ${ }^{1 *}$; COCO, M. A. ${ }^{2}$; KURITA, C. M. ${ }^{2}$; BUZETTI, M. A. ${ }^{2}$; FERREIRA-LIMA, W. ${ }^{3}$; LIMA, S. B. d. S. ${ }^{3}$

${ }^{1}$ Doutora em Ciências do Movimento Humano, Universidade Metodista de Piracicaba. Professora na Universidade Estadual do Norte do Paraná, Jacarezinho- PR, Brasil.

${ }^{2}$ Graduação em Educação Física, Universidade Estadual do Norte do Paraná, JacarezinhoPR, Brasil.

${ }^{3}$ Doutor(a) em Atividade Física e Saúde, Universidade de Extremadura, Cáceres, Espanha.

*Autora correspondente: Flávia Évelin Bandeira Lima - E-mail: flavia.lima@uenp.edu.br

DOI: https://doi.org/10.53817/1983-6929-ragg-v12n1-3 


\section{RESUMO}

Introdução: Dentre as mudanças que ocorrem durante o processo de envelhecimento, a diminuição de massa magra e aumento de gordura corporal são fatores que podem afetar seriamente a saúde do idoso. Objetivo: Analisar os efeitos da ginástica aeróbia sobre as variáveis antropométricas de idosos. Metodologia: Realizou-se um estudo de abordagem quantitativa, de caráter descritivo e comparativo e do tipo experimental. A amostra foi composta por 140 idosos, sendo 20 do sexo masculino e 120 do sexo feminino, praticantes de ginástica aeróbica, que participavam de um programa regional de atividade física para a terceira idade, na cidade de Ribeirão do Pinhal - PR. A mensuração das medidas antropométricas foi realizada em duas fases, com um período de intervalo de três meses. Foi verificado peso, estatura, aferição da pressão arterial e dos batimentos cardíacos, aplicado o teste de aptidão física em idosos (TAFI), realizado o cálculo de índice de massa corpórea (IMC) e relação cintura quadril (RCQ) de todos os participantes. Resultados: Os resultados indicaram diferença significativa nos componentes de saúde, como a massa corporal, IMC, cintura, quadril, flexibilidade, pressão arterial sistólica e diastólica. Conclusão: Com base nos resultados obtidos, observou-se que houve melhora significativa nos componentes antropométricos dos idosos.

Palavras-chave: Saúde do Idoso. Aptidão Física Funcional. Composição corporal.

\section{ABSTRACT}

Introduction: Among the changes that occur during the aging process, the decrease in lean mass and increase in body fat are factors that can seriously affect the health of the elderly. Objective: To analyze the effects of aerobic gymnastics on the anthropometric variables of the elderly. Methodology: A quantitative, descriptive and comparative study and an experimental study was carried out. The sample consisted of 140 elderly people, 20 males and 120 females, practicing aerobic gymnastics, who participated in a regional physical activity program for the elderly, in the city of Ribeirão do Pinhal - PR. The measurement of anthropometric measurements was carried out in two phases, with an interval of three months. Weight, height, blood pressure and heart rate were checked, the physical fitness test was applied to the elderly (TAFI), and the body mass index (BMI) and waist-to-hip ratio (WHR) were calculated for all participants. Results: The results indicated a significant difference in health components, such as body mass, BMI, waist, hip, flexibility, systolic and 
diastolic blood pressure. Conclusion: Based on the results obtained, it was observed that there was an improvement in the anthropometric components of the elderly.

Keywords: Health of the Elderly. Functional Fitness. Body Composition.

\section{INTRODUÇÃO}

Ao longo dos últimos anos, com o avanço da medicina e a alta da procura em relação à prática de atividades físicas, a expectativa de vida da população tem aumentado se comparado com as últimas cinco décadas, melhorando sua qualidade de vida e assim também a longevidade (FERRETTI et al., 2015). Com base nesses dados, o processo de envelhecimento, bem como suas consequências, vem sendo amplamente explorado pela comunidade científica (MORAES, 2018).

Dentre as mudanças que ocorrem durante o processo de envelhecimento, a diminuição de massa magra e o aumento de gordura corporal são fatores que podem afetar seriamente a saúde do idoso, tornando extremamente importante a avaliação e a melhora dos componentes antropométrico (MENEZES et al., 2013). Essas mudanças estão geralmente associadas à diminuição dos níveis de aptidão física, o que resulta no declínio das capacidades funcionais (COSTA et al., 2017). Dentro desse contexto, a atividade física desempenha um papel essencial na manutenção da saúde e no controle das mudanças causadas pelo envelhecimento, promovendo melhor qualidade de vida para esses indivíduos (BURTON et al., 2017).

Os benefícios descritos pelo exercício físico na funcionalidade e composição corporal de idosos são inúmeros, como melhora da flexibilidade; fortalecimento do tecido conjuntivo; manutenção ou incremento da massa muscular; força muscular; densidade óssea; e controle ou diminuição da gordura corporal (MATSUDO et al., 2000; NELSON et al., 2007). Portanto, uma prática adequada de atividades físicas, mesmo considerada tardia, se iniciada na terceira idade, promove inúmeros benefícios ao idoso (BORGES e MOREIRA, 2009).

Nesse sentido, as modificações funcionais e morfológicas relacionadas ao envelhecimento constituem uma das maiores preocupações para os profissionais da área da saúde, principalmente no que se refere à qualidade de vida e prevenção de doenças (ROBERGS e ROBERTS, 2002). Estudos afirmam que a prática regular de exercício físico pode ajudar e contribuir para o controle da depressão e diminuição da ansiedade no idoso, o que possibilita ao mesmo maior adaptação ao passar dos anos, com seu corpo e funções (ASSIS, 2004). 
Devido à escassez de estudos na literatura que evidenciam os benefícios do treinamento aeróbio para as variáveis antropométricas de idosos, o presente estudo tem como objetivo analisar os efeitos da ginástica aeróbica sobre as variáveis antropométricas de idosos.

\section{MÉTODOS}

Realizou-se um estudo de abordagem quantitativa, de caráter descritivo e comparativo e do tipo experimental, realizado com idosos, participantes do projeto "Melhor Idade", que tem ação conjunta com o Centro de Referência de Assistência Social (CRAS) e prefeitura do município de Ribeirão do Pinhal-PR.

Participaram do estudo 140 indivíduos, sendo 20 do sexo masculino e 120 do sexo feminino, com idade acima de 60 anos, todos praticantes de ginástica aeróbica. Como critérios de inclusão, foram selecionados apenas aqueles com idade igual ou acima de 60 anos, que participavam regularmente do projeto "Melhor Idade" e retornaram com o Termo de Consentimento Livre e Esclarecido devidamente assinado.

As aulas eram realizadas três vezes por semana, com duração de 60 minutos e sempre ministradas pelo professor contratado pelo município. O treinamento proposto aos idosos buscava a melhora da saúde mental, flexibilidade, força muscular e aptidão cardiorrespiratória. $\mathrm{O}$ intervalo entre a coleta de dados pré e pós-intervenção foi de três meses.

Utilizou-se uma balança digital com antropômetro da marca Welmy para verificar o peso e a estatura. Os participantes foram pesados em pé, vestindo roupas leves e sem sapatos. A estatura foi medida utilizando fita antropométrica inextensível, marca Seca®, com variação em milímetros, fixada a parede desprovida de rodapé. As circunferências da cintura e do quadril foram medidas com fita antropométrica inextensível, marca Cescorf®, com variação em milímetros.

A circunferência da cintura ( $C C)$ foi mensurada no ponto médio entre a última costela e a crista ilíaca e a circunferência do quadril (CQ), foi medida na maior circunferência da região glútea visualizada lateralmente (BRASIL, 2017; NETA et al., 2017). A relação cintura/quadril (RCQ) foi obtida a partir da divisão dos perímetros da circunferência da cintura pela circunferência do quadril. Os valores de referência como indicadores de risco para doenças cardiovasculares adotado para homens foi $\geq 0,90$ e para mulheres $\geq 0,80$ (GHARAKHANLOU et al., 2012).

A partir das medidas antropométricas de peso e estatura, foi calculado o Índice de Massa Corporal (IMC), obtido a partir da relação peso/altura². Para o diagnóstico do estado 
nutricional, foram adotados os pontos de corte propostos por Lipschitz (1994), que considera como baixo peso os valores de IMC menores que $22 \mathrm{~kg} / \mathrm{m}^{2}$, eutrófico entre 22 $\mathrm{kg} / \mathrm{m}^{2}$ e $27 \mathrm{~kg} / \mathrm{m}^{2}$, e sobrepeso acima de $27 \mathrm{~kg} / \mathrm{m}^{2}$.

A pressão arterial e os batimentos cardíacos foram mensurados através de dois aparelhos digitais da marca Omron. Aplicou-se o teste de flexibilidade do Teste de Aptidão Física em Idosos (TAFI) (SAWKA et al., 2007). Para o teste de flexibilidade, foi instruído que cada participante posicionar-se sentado no meio do assento de uma cadeira com 40 $\mathrm{cm}$ de altura, deixasse a perna esquerda semiflexionada e a perna direita estendida, com a ponta do pé voltada para cima, em seguida, ao se sentir relaxado o suficiente, inclinaria o tronco para frente estendendo o braço direito e tocando a ponta dos dedos do pé direito com a mão. Se o indivíduo não conseguisse atingir a meta, seria medido o espaço entre a ponta do dedo médio da mão até o dedo do pé direito, como consequência esta medida seria negativa indicando os centímetros que faltaram. Ao conseguir tocar o dedo do pé com o dedo médio da mão, a medida era considerada suficiente e demonstrada com o número 0 (zero), e se conseguissem ultrapassar o dedo médio da mão através do dedão de seu pé, essa medida era considerada positiva, onde foram medidos os centímetros da ponta do dedo maior do pé até a ponta do dedo médio da mão. O teste de flexibilidade foi realizado três vezes em cada voluntário, sendo utilizada apenas a melhor das três medidas.

A intervenção foi realizada em dois momentos, com um intervalo de três meses entre as coletas. A primeira fase teve início no dia 2 de março de 2018 e foi encerrada no dia 28 de março de 2018 e a segunda fase teve início no dia 18 de junho de 2018 e se encerrou no dia 13 de julho de 2018. Os participantes realizam aula de ginástica aeróbia, com duração de 60 minutos no total, distribuídas em 20 minutos de aquecimento, 35 minutos de parte especifica e 5 minutos de volta à calma. As aulas eram realizadas três vezes por semana (segunda-feira, quarta-feira e sexta-feira), sempre ministradas pelo professor contratado pelo município. O treinamento proposto aos idosos buscava a melhora da saúde mental, flexibilidade, força muscular e aptidão cardiorrespiratória.

Após a coleta de dados, a análise estatística foi realizada através do Statistical Package for the Social Science (SPSS), versão 26.0. Para a análise das variáveis numéricas recorreu-se aos procedimentos da estatística descritiva (frequência absoluta e relativa), posteriormente, para verificar a normalidade dos dados, utilizou-se o teste Kolmogorov-Smirnov. Tendo em vista a normalidade dos dados, para a comparação em dois momentos realizou-se o teste de Wilcoxon. O nível de significância adotado foi de $p \leq$ 0,05 .

\section{RESULTADOS}


A Tabela 1 apresenta a caracterização dos valores pré e pós testes, das medidas antropométricas e variação hemodinâmica de idosos ativos treinados, praticantes de ginástica aeróbica, com frequência de três vezes semanais. Realizou-se a comparação pré e pós testes e verificou-se diferenças significativas em sete variáveis, $\operatorname{IMC}(p<0,001)$, cintura $(p<0,001)$, quadril $(p<0,001)$, flexibilidade $(p<0,001)$, pressão arterial sistólica $(p=0,023)$ e pressão arterial diastólica $(p=0,002)$.

Tabela 1- Comparação pré e pós testes das medidas antropométricas, pressão arterial e batimentos por minuto de idosos praticantes de ginástica aeróbica.

\begin{tabular}{|c|c|c|c|}
\hline Variáveis & $\begin{array}{c}\text { Pré } \\
\text { Média } \pm D P\end{array}$ & $\begin{array}{c}\text { Pós } \\
\text { Média } \pm D P\end{array}$ & $\begin{array}{c}\text { Wilcoxon } \\
\text { p-valor }\end{array}$ \\
\hline Massa Corporal & $69,657 \pm 10,9302$ & $69,01 \pm 10,859$ & $<0,001^{*}$ \\
\hline Estatura & $1,5413^{a} \pm, 07843$ & $1,5413^{a} \pm, 07843$ & 1,000 \\
\hline IMC & $29,3436 \pm 4,26383$ & $29,0794 \pm 4,28331$ & $<0,001^{*}$ \\
\hline Cintura & $97,69 \pm 10,743$ & $95,71 \pm 10,591$ & $<0,001^{*}$ \\
\hline Quadril & $101,61 \pm 9,241$ & $99,45 \pm 9,014$ & $<0,001^{*}$ \\
\hline$R C Q$ &, $9627 \pm, 07484$ & ,9636, \pm 07832 & 0,507 \\
\hline Flexibilidade & $1,69 \pm 7,773$ & $4,42 \pm 8,038$ & $<0,001^{*}$ \\
\hline PAS mmHg & $145,34 \pm 24,463$ & $138,7 \pm 17,860$ & $0,023^{*}$ \\
\hline$P A D \mathrm{mmHg}$ & $83,99 \pm 12,890$ & $79,81 \pm 9,697$ & $0,002^{*}$ \\
\hline$B P M$ & $77,34 \pm 13,635$ & $77,46 \pm 10,164$ & 0,873 \\
\hline
\end{tabular}

Legenda: IMC: Índice de Massa Corporal; RQC: Razão Cintura/Quadril; DP: Desvio Padrão; PAS: Pressão Arterial Sistólica; PAD: Pressão Arterial Diastólica; BPM: Batimentos por Minuto; *Diferença significativa entre os grupos; $p<0,05$.

Fonte: Elaborado pelos autores. Dados da pesquisa.

\section{DISCUSSÃO}

Mudanças na composição corporal relacionadas ao aumento da idade podem explicar alterações no funcionamento físico e na capacidade de trabalho, visto que idosos tendem a ter maior gordura corporal e menos massa magra (BRADY et al., 2014; KENNY et al., 2016). Os idosos do presente estudo classificam-se com excesso de peso, porém houve uma redução estatisticamente significativa desse valor no resultado pós-intervenção. Um estudo que buscou avaliar o perfil nutricional e antropométrico de idosos praticantes de ginástica (FARINEA et al., 2010) também constatou valores inadequados de IMC, porém não foram feitas avaliações pré e pós-intervenção. Diferentemente dos achados por um estudo que verificou os efeitos do pilates e do exercício resistido sobre a obesidade central e o índice de massa corpórea em idosos (PESTANA et al., 2012), onde os participantes também foram classificados com sobrepeso e ocorreu aumento do IMC pós intervenção, 
porém acredita-se que esse resultado esteja relacionado ao aumento de massa muscular e não de gordura corporal.

Verificou-se no presente estudo uma melhora significativa nos componentes de cintura e quadril, corroborando, um estudo que avaliou os aspectos nutricionais de idosos praticantes de vôlei, hidroginástica e ginástica aeróbica (DÓREA et al., 2015) também encontrou diferenças significativas nesses componentes, mas apenas nos participantes do sexo feminino. Em contrapartida, em uma análise da composição corporal e do perfil lipídico de idosos praticantes de ginástica aeróbica (RICARDO, 2013), não só essas variáveis, mas também o IMC, não apresentaram diferenças significativas pré e pós-intervenção.

O envelhecimento pode ser uma das causas da diminuição dos níveis de flexibilidade, tornando importante a prática de atividade física para manutenção e melhora desse componente (PETREÇA et al., 2011). Os idosos do presente estudo apresentaram melhora significativa nesse componente após a intervenção, corroborando com os achados de um estudo que verificou o efeito do método Pilates na flexibilidade, qualidade de vida e nível de dor de idosos (TOZIM et al., 2014), onde após 8 semanas de prática também foram encontradas diferenças significativas nesse componente. Esses resultados podem estar diretamente relacionados com o ganho de força que a ginástica aeróbica e o Pilates promovem, visto que o mesmo influencia em níveis satisfatórios de flexibilidade (PETREÇA et al., 2011).

Além dos prejuízos musculares, o processo de envelhecimento também acarreta alterações no sistema cardiovascular, alterações essas que podem ocorrer mesmo na ausência de doenças e provocar aumento da pressão arterial (MENDES e BARATA, 2008; BRANDÃO et al., 2010). Nesta pesquisa observou-se melhora significativa na PAS e PAD dos idosos, concomitante ao estudo que verificou o efeito hipotensivo do treinamento de força em homens idosos (MUTTI et al., 2010), encontrando melhoras nessas variáveis.

Não foram encontradas diferenças significativas nos componentes estatura, o que pode estar relacionado ao fator biológico e RCQ. Em estudo realizado para verificar indicadores antropométricos do estado nutricional de idosas praticantes e não praticantes de exercícios físicos (CACHONI et al., 2011), realizou-se uma comparação entre os grupos e não foi verificado diferenças significativas no componente RCQ.

A Frequência Cardíaca de pessoas idosas se recupera lentamente após o exercício, o que pode representar um aumento no risco de mortalidade (ALMEIDA e ARAÚJO, 2003), corroborando, o presente estudo não obteve resultados significativos nos dados de BPM após a intervenção. Em contrapartida, em estudo realizado para verificar os efeitos do treinamento aeróbio e resistido nas respostas cardiovasculares de idosos ativos (LOCKS et al., 2012), verificou-se uma melhora na recuperação da FC em repouso. 
Uma das limitações do presente estudo pode ter sido a não aferição das dobras cutâneas dos idosos e a falta de acompanhamento nutricional. Sugere-se, outros estudos que, além das variáveis aqui analisadas, atente-se à aferição das dobras cutâneas e o acompanhamento nutricional dos idosos.

\section{CONCLUSÃO}

Com base nos resultados obtidos, observou-se melhora significativa nos componentes antropométricos dos idosos praticantes de ginástica aeróbica pré e pósintervenções, porém, o número de indivíduos com sobrepeso e excesso de peso foi muito alto, mostrando dados preocupantes para a saúde física dos participantes. Sendo assim, é necessário que esses indivíduos tenham um acompanhamento por parte dos professores envolvidos com as aulas propostas, evidenciando a importância da prática regular e sistematizada de atividade física nessa fase da vida.

Apesar das limitações metodológicas inerentes, consideramos este estudo relevante para a contribuição e incentivo de profissionais que venham a trabalhar com essa população, principalmente em programas para a comunidade, para que planejem suas aulas de acordo com as necessidades dos participantes, visando não só o prazer dos mesmos durante as aulas, mas também a melhora dos componentes antropométricos e da aptidão física voltada à saúde.

\section{REFERÊNCIAS}

ALMEIDA, M. B.; ARAÚJO, C. G. S. Efeitos do treinamento aeróbico sobre a freqüência cardíaca. Revista Brasileira de Medicina do Esporte, v. 9, n. 2, p. 104-112, 2003.

ASSIS, M. D. Aspectos sociais do envelhecimento. Em: SALDANHA, A. L.; CALDAS, C. P. (Eds.): Saúde do idoso: a arte de cuidar, $2^{a}$ Ed. Rio de Janeiro: Interciência, p. 11-21, 2004.

BORGES, M. R. D.; MOREIRA, Â. K. Influências da prática de atividades físicas na terceira idade: estudo comparativo dos níveis de autonomia para o desempenho nas AVDs e AIVDs entre idosos ativos fisicamente e idosos sedentários. Motriz, Revista de Educação Física, UNESP, v. 15, n. 3, p. 562-573, 2009.

BRADY, A. O.; STRAIGHT, C. R.; EVANS, E. M. Body composition, muscle capacity, and physical function in older adults: an integrated conceptual model. Journal of aging and physical activity, v. 22, n. 3, p. 441-452, 2014.

BRANDÃO, A. A. et al. VI diretrizes brasileiras de hipertensão. Arquivos Brasileiros de Cardiologia, v. 95, n. 1, p.1-51, 2010. 
Brasil. Ministério da Saúde. Caderneta de Saúde da Pessoa Idosa. 4aㅡ ed. Brasília: DF; 2017. 61 p. Disponível em:

http://portalarquivos2.saude.gov.br/images/pdf/2017/setembro/27/CADERNETA-PESSOAIDOSA-2017-Capa-miolo.pdf

BURTON, E. et al. Why do seniors leave resistance training programs? Clinical Interventions in Aging, v. 12, p. 585, 2017.

CACHONI, L. et al. Indicadores antropométricos do estado nutricional de idosas praticantes e não praticantes de exercício físico na zona norte de São José do Rio Preto-SP. Alimentos e Nutrição Araraquara, v. 21, n. 4, p. 537-546, 2011.

COSTA, K. M. S. M. et al. Perfil antropométrico, funcional e cognitivo de idosos não institucionalizados. Revista Brasileira de Pesquisa em Ciências da Saúde, v. 3, n. 2, p. 28-35, 2017.

DÓREA, G. D. S.; MANOCHIO-PINA, M. G.; SANTOS, D. D. Aspectos nutricionais de idosos praticantes de atividade física. DEMETRA: Alimentação, Nutrição \& Saúde, v. 10, n. 2, p. 347-360, 2015.

FARINEA, N.; RICALDE, S. R.; SIVIERO, J. Perfil nutricional e antropométrico de idosos participantes de um grupo de ginástica no município de Antônio Prado-RS. Revista Brasileira de Ciências do Envelhecimento Humano, v. 7, n. 3, p. 394-405, 2010.

FERRETTI, F. et al. Análise da qualidade de vida em idosos praticantes e não praticantes de exercício físico regular. Estudos Interdisciplinares sobre 0 Envelhecimento, v. 20, n. 3, p. 729-743, 2015.

GHARAKHANLOU, R. et al. Medidas antropométricas como preditoras de fatores de risco cardiovascular na população urbana do Irã. Arquivos Brasileiros de Cardiologia, v. 98, n. 2, p. 126-135, 2012.

KENNY, G. P. et al. Age, human performance, and physical employment standards. Applied physiology, nutrition, and metabolism, v. 41, n. 6, p. S92-S107, 2016.

LIPSCHITZ, D. A. Screening for nutritional status in the elderly. Primary care, v. $21, \mathrm{n}$. 1, p. 55-67, 1994.

LOCKS, R. R. et al. Efeitos do treinamento aeróbio e resistido nas respostas cardiovasculares de idosos ativos. Fisioterapia em Movimento, v. 25, n. 3, p. 541-550, 2012.

MATSUDO, S. M.; MATSUDO, V. K. R.; NETO, T. L. B. Efeitos benéficos da atividade física na aptidão física e saúde mental durante o processo de envelhecimento.

Revista Brasileira de Atividade Física \& Saúde, v. 5, n. 2, p. 60-76, 2000.

MENDES, R.; BARATA, J. T. Envelhecimento e pressão arterial. Acta Médica Portuguesa, v. 21, n. 2, p. 193-198, 2008.

MENEZES, T. N. D. et al. Perfil antropométrico dos idosos residentes em Campina Grande-PB. Revista Brasileira de Geriatria e Gerontologia, v. 16, n. 1, p. 19-27, 2013.

MORAES, L. P. d. Comparação dos efeitos do treinamento resistido e da hidroginástica na percepção de qualidade de vida e IMC de indivíduos idosos. 2018. 
89 p. Dissertação (Mestrado em Atividade Física para a Terceira Idade). Faculdade de Desporto, Universidade do Porto, Portugal. Disponível em:

https://hdl.handle.net/10216/116905. Acesso em: 06 jul. 2020.

MUTTI, L. C. et al. Efeito hipotensivo do treinamento de força em homens idosos. Revista Brasileira de Cardiologia, v. 23, n. 2, p. 111-115, 2010.

NELSON, M. E. et al. Physical activity and public health in older adults:

recommendation from the American College of Sports Medicine and the American Heart Association. Circulation, v. 116, n. 9, p. 1094, 2007.

NETA, A. d. C. P. d. A. et al. Índice de conicidade como preditor de alterações no perfil lipídico em adolescentes de uma cidade do Nordeste do Brasil. Cadernos de Saúde Pública, v. 33, n. 3, p. e00029316, 2017.

PESTANA, V. S. et al. Efeitos do Pilates solo e exercício resistido sobre a obesidade central e o índice de massa corpórea em idosos. Revista de Ciências Médicas e Biológicas, v. 11, n. 2, p. 218-223, 2012.

PETREÇA, D. R.; BENEDETTI, T. R. B.; SILVA, D. A. S. Validação do teste de flexibilidade da AAHPERD para idosos Brasileiros. Revista Brasileira de Cineantropometria \& Desempenho Humano, v. 13, n. 6, p. 455-460, 2011.

RICARDO, G. S. Análise da composição corporal e do perfil lipídico de praticantes de ginástica aeróbia. 2013. 49 p. Monografia (Trabalho de Conclusão de Curso em Educação Física Bacharelado). Universidade do Extremo Sul Catarinense, Criciúma-SC.

ROBERGS, R. A.; ROBERTS, S. O. Princípios fundamentais de fisiologia do exercício para aptidão, desempenho e saúde. Em: ROBERGS, R.A.; ROBERTS, S. O. (Eds.): Princípios fundamentais de fisiologia do exercício para aptidão, desempenho e saúde, 1ํㅡㄹ Ed. São Paulo: Phorte, p. 511-511, 2002.

SAWKA, M. N. et al. American College of Sports Medicine position stand. Exercise and fluid replacement. Medicine and science in sports and exercise, v. 39, n. 2, p. 377390, 2007.

TOZIM, B. M. et al. Efeito do método Pilates na flexibilidade, qualidade de vida e nível de dor em idosos. ConScientiae Saúde, v. 13, n. 4, p. 563-570, 2014. 